\title{
Diversidad del género Dahlia (Asteraceae: Coreopsideae) en Jalisco, México y descripción de una especie nueva
}

\section{Diversity of the genus Dahlia (Asteraceae: Coreopsideae) in Jalisco, Mexico and description of a new species}

\author{
Arturo Castro-Castro, Aarón Rodríguez ${ }^{\bigotimes}$, Georgina Vargas-Amado y Mollie Harker \\ Centro Universitario de Ciencias Biológicas y Agropecuarias, Departamento de Botánica y Zoología, Universidad de Guadalajara. Apartado postal \\ 1-139, 45101 Zapopan, Jalisco, México. \\ \rca08742@cucba.udg.mx
}

\begin{abstract}
Resumen. La tribu Coreopsideae Turner et Powell (Asteraceae) concentra su diversidad y endemismo en México. Dahlia Cav. es un género característico de Coreopsideae; en Jalisco crecen D. barkerae Knowles et Westc., D. coccinea Cav., D. pugana Aaron Rodr. et A. Castro, D. sherffii P. D. Sorensen y D. tenuicaulis P. D. Sorensen. Aquí se describe e ilustra D. pugana, una especie nueva para la ciencia. Hasta donde sabemos, este taxón es exclusivo de Jalisco, pertenece a Dahlia sección Dahlia y tiene semejanzas morfológicas con D. barkerae, D. brevis P. D. Sorensen y D. scapigera (A. Dietr.) Knowles et Westc. con las cuales se compara. Por último, incluimos una clave para la identificación de las especies que crecen en Jalisco.

Palabras clave: Compositae, Heliantheae, endemismo, riqueza.

Abstract. The greatest diversity and endemism of Coreopsideae Turner et Powell (Asteraceae) is concentrated in Mexico. Dahlia Cav. is a characteristic genus of Coreopsideae and represented in Jalisco by D. barkerae Knowles et Westc., D. coccinea Cav., D. pugana Aaron Rodr. et A. Castro, D. sherffii P. D. Sorensen and D. tenuicaulis P. D. Sorensen. We describe and illustrate D. pugana as a new species. As far as we know, this taxon is exclusive to Jalisco, pertains to Dahlia section Dahlia, and has morphological similarities with D. barkerae, D. brevis P. D. Sorensen and D. scapigera (A. Dietr.) Knowles et Westc. with which it is compared. Finally, we include an identification key for the species that grow in Jalisco.
\end{abstract}

Key words: Compositae, Heliantheae, endemism, species richness.

\section{Introducción}

El occidente mexicano es un centro de diversidad de la familia Asteraceae (Villaseñor et al., 2005). Un ejemplo de la riqueza y endemismo de las asteráceas es la tribu Coreopsideae Turner et Powell, integrada por 24 géneros y 590 especies, de las cuales alrededor de 80 (15\%) son exclusivas de México (Crawford et al., 2009). Una región de singularidades botánicas en México es la zona de confluencia entre el extremo oeste del Eje Volcánico Transversal, el límite sur de la sierra Madre Occidental y la porción septentrional de la sierra Madre del Sur en el estado de Jalisco. En esta zona crecen 8 especies (10\%) de las Coreopsideae endémicas del país.

En Jalisco, el género Dahlia Cav. (Coreopsideae) es conocido coloquialmente como "dalias", "charahuescas" o "camilas"; sus numerosos cultivares se han desarrollado

Recibido: 10 mayo 2011; 17 octubre 2011 desde tiempos prehispánicos, tienen gran aceptación como ornamentales y $D$. coccinea Cav. se considera como la flor nacional de México. Las dalias son plantas perennes con raíces tuberosas y tallos herbáceos o lignificados. La mayoría son herbáceas o arbustos, mientras que $D$. macdougallii Sherff es una hierba epífita (Sherff, 1955; Sorensen, 1969; Cuadro 1). Las dalias crecen con mayor frecuencia en bosques de encino o de encino y pino, entre los 1500 y los $3700 \mathrm{~m} \mathrm{snm}$. Sin embargo, D. coccinea también se encuentra en bosques tropical caducifolio, subcaducifolio y perennifolio y en elevaciones cercanas al nivel del mar.

Dahlia ha sido sujeto de diversos estudios taxonómicos y filogenéticos. Sorensen (1969) reconoció 27 taxones agrupados en 4 secciones: Dahlia sección Dahlia, Dahlia sección Entemophyllon P. D. Sorensen, Dahlia sección Epiphytum Sherff y Dahlia sección Pseudodendron P. D. Sorensen. Sin embargo, trabajos recientes reconocen en el grupo a 37 especies (Sherff, 1946; Sorensen, 1980, 1987; Saar y Sorensen, 2000, 2005; Saar, 2002; Saar et al., 2002, 
Cuadro 1. Diversidad y distribución geográfica de Dahlia en México. La clasificación es la de Sorensen (1969)

\begin{tabular}{|c|c|c|}
\hline Sección & Especie & Distribución \\
\hline \multirow[t]{25}{*}{ Dahlia } & D. apiculata P. D. Sorensen & Oax, Pue \\
\hline & D. atropurpurea P. D. Sorensen & Gro, Méx \\
\hline & D. australis (Sherff) P. D. Sorensen & Chis, Oax \\
\hline & D. barkerae Knowles et Westc. & Jal, Mich \\
\hline & D. brevis P. D. Sorensen & Méx, Mich \\
\hline & D. coccinea Cav. & México excepto B.C., B.C.S., Yuc, Q.R., Camp \\
\hline & D. cordifolia (Sessé et Moc.) McVaugh & Gro \\
\hline & D. cuspidata D. E. Saar, P. D. Sorensen et J. P. Hjert. & Gto, Hgo, Qro \\
\hline & D. hintonii Sherff & Gro \\
\hline & D. hjertingii H. V. Hansen et P. D. Sorensen & Hgo \\
\hline & D. merckii Lehm. & $\begin{array}{c}\text { Méx, D.F., Gto, Gro, Hgo, Mich, Mor, N.L., Oax, Pue, } \\
\text { Qro, S.L.P., Tamps, Ver }\end{array}$ \\
\hline & D. mollis P. D. Sorensen & Gto, Hgo, Qro \\
\hline & D. moorei Sherff & Hgo, Qro \\
\hline & D. neglecta D. E. Saar & D. F., Méx, Gto, Hgo, Mich, Qro, Ver \\
\hline & D. parvibracteata D. E. Saar et P. D. Sorensen & Gro \\
\hline & D. pteropoda Sherff & Oax, Pue \\
\hline & *D. pugana Aaron Rodr. et A. Castro & Jal \\
\hline & *D. purpusii Brandegee & Chis \\
\hline & D. rudis P. D. Sorensen & D.F., Méx, Mich, Mor \\
\hline & D. scapigera (A. Dietr.) Knowles et Westc. & D.F., Méx, Gto, Hgo, Mich \\
\hline & D. sherffii P. D. Sorensen & Chih, Dgo, Jal \\
\hline & D. sorensenii H. V. Hansen et J. P. Hjert. & D.F., Méx, Hgo, Mich, Qro, S.L.P. \\
\hline & *D. spectabilis D. E. Saar, P. D. Sorensen et J. P. Hjert. & S.L.P. \\
\hline & D. tenuis B. L. Rob. et Greenm. & Oax \\
\hline & D. tubulata P. D. Sorensen & N.L., Tamps \\
\hline \multirow[t]{7}{*}{ Entemophyllon } & *D. congestifolia P. D. Sorensen & Hgo \\
\hline & D. dissecta $\mathrm{S}$. Watson & Hgo, Qro, S.L.P., Tamps \\
\hline & D. foeniculifolia Sherff & N.L., Tamps \\
\hline & D. linearis Sherff & Gto, Qro \\
\hline & D. rupicola P. D. Sorensen & Dgo \\
\hline & D. scapigeroides Sherff & Gto, Hgo, Qro, S.L.P. \\
\hline & D. sublignosa (P. D. Sorensen) D. E. Saar et P. D. Sorensen & Tamps \\
\hline Epiphytum & *D. macdougallii Sherff & Oax \\
\hline \multirow[t]{4}{*}{ Pseudodendron } & D. campanulata D. E. Saar, P. D. Sorensen et J. P. Hjert. & Oax \\
\hline & D. excelsa Benth. & Méx, Gro \\
\hline & D. imperialis Roezl ex Ortgies & Chis, Oax \\
\hline & D. tenuicaulis P. D. Sorensen & Gro, Jal, Mich, Oax \\
\hline
\end{tabular}

*Especie conocida sólo de su localidad tipo. 
2003b; Hansen y Sorensen, 2003; Hansen, 2004). Por otro lado, Saar et al. (2003a), encontraron que sólo Dahlia sección Entemophyllon forma un clado natural, mientras que la monofilia de las secciones Dahlia, Epiphytum y Pseudodendron está en discusión.

\section{Materiales y métodos}

El trabajo se realizó en 3 fases. La primera consistió en la búsqueda, obtención y revisión de literatura; la segunda fase comprendió la revisión de los herbarios que se enumeran a continuación y la determinación de ejemplares de Dahlia depositados en ellos: Herbario del Centro Interdisciplinario para el Desarrollo Integral Regional-Michoacán (CIMI); Herbario del Centro Interdisciplinario para el Desarrollo Integral RegionalDurango (CIIDIR); Herbario de la Universidad Autónoma de Guadalajara (GUADA), Herbario Luz María Villarreal de Puga, Instituto de Botánica de la Universidad de Guadalajara (IBUG), Herbario Graciela Calderón y Jerzy Redowski, Centro Regional del Bajío, Instituto de Ecología A. C. (IEB) y Herbario Nacional de México, Instituto de Biología, Universidad Nacional Autónoma de México (MEXU); además, se revisaron fotografías digitalizadas de ejemplares depositados en las siguientes instituciones: New York Botanical Garden Herbarium (NY), Rancho Santa Ana Botanic Garden Herbarium (RSA) y United States National Herbarium, Department of Botany, Smithsonian Institution (US). Con la información obtenida de los ejemplares de herbario se elaboró una base de datos taxonómicos, geográficos, ecológicos y curatoriales. Por último, la tercera fase incluyó el trabajo de campo, con numerosos viajes de exploración y recolección de las especies de Dahlia, principalmente en Jalisco.

\section{Resultados}

Con base en la información de 337 ejemplares de herbario y la consulta de diversas publicaciones (Sorensen, 1969; Sorensen, 1980; McVaugh, 1984; Sorensen, 1987; Bauml, 1994; Saar y Sorensen, 2000; Saar, 2002; Saar et al., 2002; Hansen y Sorensen, 2003; Saar et al., 2003a; Hansen, 2004; Saar y Sorensen, 2005) se documenta la presencia de 37 especies de Dahlia en México, que es el centro de diversificación del género (Cuadro 1). El mayor número de especies se concentra en la región centro oriental del país, en los estados de Hidalgo (11 spp.), Querétaro (10 spp.), Estado de México (9 spp.), Michoacán (9 spp.), Oaxaca (9 spp.), Guanajuato (8 spp.) y Guerrero (7 spp.). Dahlia australis (Sherff) P. D. Sorensen, D. coccinea y D. imperialis Roezl ex Ortgies se extienden hacia Centroamérica y el norte de América del Sur, donde quizás fueron introducidas. Los resultados obtenidos documentan la presencia de 5 especies de Dahlia en Jalisco. Además de $D$. coccinea y D. tenuicaulis $P$. D. Sorensen, citadas por McVaugh (1984), se añaden D. barkerae Knowles et Westc., D. sherffii P. D. Sorensen y D. pugana especie que aquí proponemos como nueva para la ciencia.

Dahlia Cav. Icon. 1(3): 56-57. 1791.

Plantas herbáceas, arbustivas o epífitas, perennes; raíces subterráneas tuberosas, engrosadas y fasciculadas; tallos erectos, por lo general sin ramificarse excepto en la inflorescencia o a veces desde la base; hojas opuestas o verticiladas, simples, bipinnadas o tripinnadas, estipelas en ocasiones presentes; cabezuelas campanuladas a hemisféricas, solitarias o en panículas; pedúnculos largos; involucro biseriado; filarias externas (4) 5 (7), verdes, suculentas, erectas, extendidas o reflejas en la antesis; filarias internas (7) 8 (9), membranáceas, elíptico ovadas a obovadas, nervaduras de color amarillo, pardo, rojo o púrpura, márgenes escariosos, hialinos; páleas escariosas y parecidas a las filarias internas; flores liguladas 8, estériles o fértiles, blancas, violadas, amarillas, anaranjadas, rojas o purpúreas; flores del disco hermafroditas, numerosas, fértiles, tubulares, amarillas, rojas o moradas; ramas del estilo linear-lanceoladas y dorsalmente aristadas; filamentos glabros; anteras de ápices agudos y bases obtusas; ramas del estilo subuladas y pubescentes en el dorso; aquenios lineares, oblanceolados, espatulados, obovados o elípticos, ápices truncados, negruzcos; vilano disciforme, con 2 hombros diminutos, o bien, ausente; receptáculo plano, rara vez convexo.

Se presenta una clave para la determinación de las especies que crecen en Jalisco y a continuación las descripciones morfológicas y discusiones taxonómicas relativas a las mismas.

A. Filarias externas del involucro extendidas o reflejas en antesis.

B. Flores liguladas rojas, anaranjadas, amarillas o de color vino tinto; plantas de amplia distribución.....D. coccinea B. Flores liguladas violadas; plantas con distribución restringida.

C. Plantas herbáceas, erectas, de 0.6-1.3 m de altura con tallos no lignificados; crecen en el extremo norte

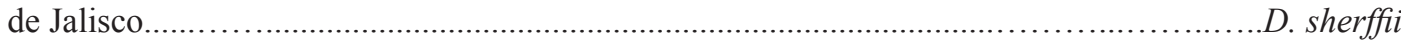

C. Plantas arborescentes o arbustivas, decumbentes, de 2-4.5 m de altura, tallos lignificados, creciendo en el sur y suroeste de Jalisco.............................................................................................. . tenuicaulis

A. Filarias externas del involucro erectas o poco extendidas en antesis. 
D. Hojas sin estipelas; flores del disco 20-55; flores liguladas estériles; aquenios con vilano disciforme; plantas de 0.4 a $0.7 \mathrm{~m}$ de alto; centro de Jalisco.......................................................... pugana

D. Hojas con estipelas; flores del disco 35-60; flores liguladas fértiles; aquenios sin vilano; plantas de 0.9 a $1.5 \mathrm{~m}$ de alto; este de Jalisco

D. barkerae

\section{Descripciones}

Dahlia barkerae Knowles et Westc., Fl. Cab. 2: 28. 1838; Fl. Cab. 3: 147, t. 127. 1838 (Fig. 1A).

Herbácea perenne, $0.9-1.5 \mathrm{~m}$ de altura, el follaje por lo general concentrado en la parte basal; raíces tuberosas fasciculadas; tallo fistuloso, con pelos multicelulares concentrados en los nudos; entrenudos de 0-1 (6.5-14) cm de longitud; pecíolo sólido, de 2.5-6 (14) cm de largo, alado hacia la base; láminas foliares elípticas a oblongas en contorno general, de $16-30 \mathrm{~cm}$ de largo y $13 \mathrm{~cm}$ de ancho, todas pinnadas, en ocasiones trifolioladas o indivisas, raquis a menudo alado, estipelas presentes; folíolos (3)57(9), opuestos o alternos, los pares distantes entre sí $3-5 \mathrm{~cm}$, sobre todo el más proximal, sésiles y decurrentes, a veces con peciólulos hasta de $4 \mathrm{~cm}$ de largo, ovados, romboides o lanceolados, 3.5-6 (9.5) cm de largo, ápice agudo, base cuneada, truncada o subcordada, margen ciliado y con 1 a 5 dientes de cada lado, de textura membranácea, verdes oscuros y pubescentes en el haz, notablemente más pálidos y glabros o con algo de pubescencia a lo largo de las nervaduras en el envés; foliolo terminal más largo que los laterales; cabezuelas 2-3 (5), de 4.5-5.5 cm de diámetro, varias sincrónicas; pedúnculos de $5.5-13 \mathrm{~cm}$ de largo; involucro campanulado, glabro; filarias externas 5, elípticas a obovadas, de 5-11 mm de largo, erectas en antesis y con 3-5 nervaduras de color púrpura; filarias internas 8 , elípticas a oblongas, de 11-16 mm de largo, agudas, amarillas pardas; páleas similares a las filarias internas; flores liguladas 8 , fértiles, tubos pilosos, lígulas ovadas a elípticas, de 1.5$3.5 \mathrm{~cm}$ de largo, violadas, glabras y con 2 nervaduras marcadas; flores del disco 35-60, tubulares, de 8-10 mm de largo, amarillas, sus lóbulos extendidos o reflejos, tubo con algunos pelos diminutos en la base; anteras de 3-4 mm de largo, de color café; ramas del estilo oblanceoladas, agudas y densamente ciliadas; aquenios linear oblanceolados, 5-8 $\mathrm{mm}$ de largo, negruzcos, pubérulos; vilano ausente o en forma de hombros diminutos; receptáculo plano, 7-9 mm de diámetro.

Comentarios taxonómicos. Dahlia barkerae sólo se conocía del municipio de Morelia, Michoacán (Rzedowski y Calderón de Rzedowski, 2008). Los recientes hallazgos en Cerro Viejo, municipios de Jocotepec y Tlajomulco de Zúñiga, Jalisco, extienden su distribución aproximadamente $250 \mathrm{~km}$ hacia el oeste y aportan nuevos datos sobre las condiciones ecológicas en las que se desarrolla. Es una hierba que crece en lugares rocosos y húmedos del bosque de encino y pino con elementos del bosque mesófilo de montaña, en altitudes que van desde los 2000 hasta los 2800 metros. El número de individuos por población es reducido, por lo que se considera susceptible de desaparecer. Sorensen (1969) estableció la sinonimia entre $D$. barkerae y D. scapigera (A. Dietr.) Knowles et Westc. var. arsenei Sherff, pero Rzedowski y Calderón de Rzedowski (2008) reconocen a D. scapigera var. arsenei debido a que en una importante proporción de los individuos que revisaron, no observaron los caracteres descritos por Sorensen (1969). No obstante, aquí hemos decidido reconocer a $D$. barkerae con base en los resultados del análisis filogenético de Saar et al. (2003a).

Material estudiado. México. Jalisco: municipio de Jocotepec: ladera de exposición sur de Cerro Viejo, subiendo por La Ventina, 23.IX.1990, flor y fruto, $R$. Ramírez-Delgadillo 2284; vereda al Corral Falso, ladera sur de Cerro Viejo, 18.IX.1995, flor y fruto, J. A. Machuca 7473 (IBUG). Municipio de Tlajomulco de Zúñiga: 1.5 $\mathrm{km}$ al noroeste de Bola del Viejo y $4.6 \mathrm{~km}$ al noroeste de Huejotitán, Cerro Viejo, camino de descenso rumbo a El Molino, 10.I.2011, fruto, A. Castro-Castro y J. GonzálezGallegos 2304 (IBUG).

Dahlia coccinea Cav., Icon. 3(2): 33-34, t. 266. 1794 (Fig. 1B).

Herbácea perenne, 1-2 (3) m de altura; raíces tuberosas; tallos fistulosos, de $2.5 \mathrm{~cm}$ de diámetro, glabros o pubescentes; hojas opuestas o verticiladas; pecíolos de 4-10 (15) cm de largo, angostamente alados; láminas foliares de formas muy variadas, enteras, pinnadas 0 bipinnadas, $15-25$ (35) $\mathrm{cm}$ de largo y 10-20 $\mathrm{cm}$ de ancho, estipelas a veces presentes; folíolos ovados, romboides o lanceolados, sésiles o con peciólulos cortos, $1-16 \mathrm{~cm}$ de largo y $0.5-13 \mathrm{~cm}$ de ancho, agudos o acuminados en el ápice, cuneados, decurrentes o truncados en la base, margen aserrado, lobado o subentero, textura membranácea, de color verde oscuro y glabros, pubescentes o escábridos en el haz, pálidos, glabros a pubescentes a lo largo de las nervaduras en el envés; cabezuelas en grupos de 2-5, 8-12 $\mathrm{cm}$ de diámetro, varias sincrónicas; pedúnculos de 20-30 cm de largo, glabros; involucro campanulado; filarias externas 5, ovadas a oblongas o espatuladas, 5-15 $\mathrm{mm}$ de largo, verdes, en ocasiones pubescentes en su cara interna, extendidas o reflejas en antesis; filarias internas 8, elípticas a ovadas u oblongas, 10-22 $\mathrm{mm}$ de largo, de color amarillo 
pardo, glabras; páleas similares a las filarias internas; flores liguladas 8 , estériles, tubos pilosos o glandulares, lígulas elípticas a ovadas u obovadas, $1.5-4.5 \mathrm{~cm}$ de largo, amarillas, anaranjadas, rojas o purpúreas, pubescentes sobre las nervaduras de su cara externa; flores del disco 25-100, tubulares, de 7-10 mm de largo, amarillas; anteras de 3-4 mm de largo, de color de café; ramas del estilo oblanceoladas y pubérulas por fuera en la parte distal, agudas; aquenios linear oblanceolados, de 8-13 mm de largo, grises a negruzcos, finamente pubérulos; vilano ausente; receptáculo plano, 10-15 mm de diámetro.

Comentarios taxonómicos. Planta abundante y ampliamente distribuida en México, con excepción de las penínsulas de Yucatán y Baja California; por lo tanto, no tiene problemas de supervivencia. Habita en bosques de encino, de coníferas, bosques mesófilos de montaña, bosques tropicales caducifolios, subcaducifolios y perennifolios. A menudo también se le encuentra en la vegetación secundaria o como ruderal, en altitudes que varían entre 900 y $2850 \mathrm{~m}$. Florece de junio a noviembre. Material estudiado. México. Jalisco: municipio de Ahualulco de Mercado: camino a Piedras Bola, 1.IX.1995, flor, J. J. Reynoso-Dueñas 2907 (IBUG). Municipio de Amacueca: Km. 14-15 carretera a Tapalpa, a partir de la carretera Guadalajara-Ciudad Guzmán, 7.IX.2009, flor y fruto, A. Rodríguez et al. 5938 (ENCB, IBUG, IEB, MEXU). Municipio de Ameca: Km. 23 carretera México 70, Ameca-Mascota, 1.IX.2009, flor, A. Castro-Castro et al. 1851 (IBUG, IEB, MEXU). Municipio de Arandas: ladera de exposición norte, antenas de microondas, cerro Gordo, 8.X.1992, flor, J. J. Reynoso-Dueñas et al. 1049 (IBUG). Municipio de Atenguillo: cerro La Campana, después de El Jacal y antes de la desviación a Volcanes, 13.VIII.1994, flor y fruto, M. Cházaro y J. A. Lomelí 7418 (IBUG). Municipio de Autlán de Navarro: puerto Los Mazos, 11.X.1986, flor, S. Zamudio 4774 (IBUG, IEB). Municipio de Bolaños: aproximadamente $30 \mathrm{~km}$ al norte de Bolaños terracería a Tuxpan, 7.X.1981, flor, R. Ornelas et al. s.n. (IBUG). Municipio de Cañadas de Obregón: trayecto del rancho El Salto, 5.IX.1976, flor, L. M. Villarreal de Puga 8961 (IBUG). Municipio de Casimiro Castillo: arroyo El Tecolote, 22.IX.1988, flor y fruto, M. Anaya 30 (IBUG). Municipio de Chapala: Mesa del Ocote, 27.VII.1996, flor, A. Padilla et al. 4 (IBUG). Municipio de Chiquilistlán: brecha TapalpaChiquilistlán, 27.VIII.1987, flor, A. Rodríguez 985 (ENCB, IBUG, IEB, MEXU). Municipio de Cocula: Tierra Blanca, carretera a Barra de Navidad, 22.IX.1989, flor, R. Ramírez-Delgadillo et al. 1586 (IBUG). Municipio de Concepción de Buenos Aires: Km. 7 a Concepción de Buenos Aires, 22.VIII.1976, flor, L. M. Villareal de Puga y I. Romero 8822 (IBUG). Municipio de Cuatitlán de García Barragan: sierra de Manantlán, 20.XII.1981, fruto, L. M. Villarreal de Puga y R. González-Tamayo 11884 (IBUG). Municipio de Gómez Farías: 5 km al noreste de La Ranchería, sobre brecha a La Cofradía, 21.IX.1989, flor y fruto, J. Villa 10 (IBUG). Municipio de Guadalajara: barranca de Huentitán, 18.IX.2004, flor y fruto, M. Petite 8 (IBUG). Municipio de Huejuquilla el Alto: San Andrés Cohamiata, flor, A. Casillas s.n. (IBUG). Municipio de Ixtlahuacán de los Membrillos: $20 \mathrm{~km}$ al sur de Atequiza, cercano al río Lerma, flor y fruto, 11.IX.1976, L. M. Villarreal de Puga \& S. Carvajal 14305 (IBUG). Municipio de Jocotepec: camino de ascenso a Cerro Viejo, por el poblado de Zapotitlán de Hidalgo, 20.VIII.1986, flor, A. Rodríguez 568 (ENCB, IBUG, IEB). Municipio de La Barca: El Abrevadero, 15.X.1982, flor y fruto, R. Pantoja-Carlos 2 (IBUG). Municipio de La Huerta: $20 \mathrm{~km}$ después de la carretera a barra de Navidad, 22.VII.1975, flor, L. M. Villarreal de Puga 7570 (IBUG). Municipio de La Manzanilla de la Paz: $5 \mathrm{~km}$ al sur de La Manzanilla, carretera La Manzanilla-Mazamitla, 18.IX.2006, flor, A. Rodríguez et al. 4959 (ENCB, IBUG, IEB, MEXU). Municipio de Lagos de Moreno: La Mesa, $6 \mathrm{~km}$ adelante de Lagos de Moreno, 28.IX.1986, flor, F. J. Santana-Michel 2127 (IBUG). Municipio de Mascota: $2 \mathrm{~km}$ al noreste del rancho El Galope, 18.X.1987, flor y fruto, R. RamírezDelgadillo et al. 751 (IBUG). Municipio de Mazamitla: $6 \mathrm{~km}$ al oeste antes del poblado de Mazamitla, frente a Puerto de Cuevas, terrenos del fraccionamiento Bosque Real, 20.IX.2008, flor, A. Castro-Castro et al. 1348 (IBUG, IEB, MEXU). Municipio de Mezquitic: Km. 50, camino Bolaños-Tenzompa, al noreste de Pinos Altos, comunidad indígena de Santa Catarina, 22.IX.1987, flor, L. M. González-Villarreal 3183 (IBUG). Municipio de Ojuelos de Jalisco: Papas de Arriba, $4.5 \mathrm{~km}$ al noroeste de Guadalupe Victoria, la cañada al sur del ejido, 3.VIII.1999, flor, M. Harker y P. Carrillo-Reyes 1256 (IBUG). Municipio de Poncitlán: Los Petroglifos, base del cerro El Alfiler, al noroeste de Poncitlán, 17.VIII.1975, flor, L. M. Villarreal de Puga 7692 (IBUG). Municipio de San Cristóbal de la Barranca: $10 \mathrm{~km}$ al norte de la desviación de la carretera a Tesistán, antes de llegar al devisadero, 4.IX.1988, flor y fruto, A. FloresMacías et al. 1003 (IBUG). Municipio de San Martín Hidalgo: sierra de Quila, parte alta de las lomas al sur del río Grande por la vereda a Lagunillas, 19.VIII.1989, flor, J. J. Guerrero-Nuño 307 (IBUG). Municipio de San Miguel el Alto: Km. 14, camino San MiguelArandas, 12.IX.1988, flor, R. Ramírez-Delgadillo et al. 956 (IBUG, IEB, MEXU). Municipio de San Sebastián del Oeste: La Bufa, 18.VIII.1994, flor, J. J. ReynosoDueñas et al. 1397 (IBUG). Municipio de Santa María 
del Oro: Los Hornos, 10.VIII.1974, flor, L. M. Villarreal de Puga y I. Romero 15890 (IBUG). Municipio de Tala: a lo largo del arroyo Caliente y Los Letreros, bosque escuela La Primavera, 13.VIII.1988, flor, A. Rodríguez y J. J. Reynoso-Dueñas 1439 (ENCB, IBUG, IEB,MEXU). Municipio de Talpa de Allende: brecha que conduce de Talpa de Allende a La Cuesta, 28.VIII.1996, flor, J. J. Reynoso-Dueñas 3263 (IBUG). Municipio de Tamazula: $27 \mathrm{~km}$ de la desviación a la brecha a Manuel M. Diéguez, 19.XI.1973, flor y fruto, L. M. Villarreal de Puga 13851 (IBUG). Municipio de Tapalpa: camino a Tapalpa, La Estancia, 16.IX.1973, flor, L. M. Villarreal de Puga 5390 (IBUG). Municipio de Tecalitlán: al sur de Tecalitlán, rumbo a Pihuamo, 12.VIII.1987, flor, $R$. Ornelas et al. 1161 (IBUG, MEXU). Municipio de Tecolotlán: sierra de Quila, arroyo El Columpio, 20.IX.2008, flor y fruto, M. Harker 3834 (IBUG). Municipio de Tenamaxtlán: recorrido de Tenamaxtlán a Los Picachos, 20.VIII.1994, flor, J. A. Machuca 7189 (IBUG). Municipio de Tequila: camino al cerro de Tequila, 26.VIII.1986, flor, $R$. Ornelas 447 (IBUG). Municipio de Tlajomulco de Zúñiga: Cerro Viejo, $5 \mathrm{~km}$ al sur de San Lucas Evangelista, 18.VIII.1989, flor, R. Ramírez-Delgadillo et al. 1477 (IBUG). Municipio de Tuxpan: brecha Atenquiquevolcán de Colima, 18.III.1976, fruto, L. M. Villarreal de Puga 8448 (ENCB, IBUG). Municipio de Valle de Guadalupe: rancho Tepozanes (cerro de Ramblas), $7 \mathrm{~km}$ al oeste noroeste del valle de Guadalupe, 1.VIII.2001, flor, P. Carrillo-Reyes 2392 (IBUG). Municipio de Zapopan: carretera a San Cristóbal de la Barranca, 24.VIII.1995, flor, J. J. Reynoso-Dueñas 2852 (IBUG). Municipio de Zapotitlán: San Antonio, en el límite con el estado de Colima, 28.VIII.1982, flor, L. M. González-Villarreal 1455 (IBUG). Municipio de Zapotlanejo: Puente de Guadalupe, $5 \mathrm{~km}$ al oeste y a $75 \mathrm{~m}$ del río Santiago, 8.VII.1986, flor, M. Sánchez 10 (IBUG). Municipio de Zapotlán el Grande: puerto El Floripondio, en el Nevado de Colima, flor, A. Flores y R. Cuevas 244 (IBUG).

Dahlia sherffii P. D. Sorensen, Rhodora 71: 387. 1969 (Fig. 1C).

Herbácea perenne, 0.6-1.3 m de altura; raíces tuberosas; tallos fistulosos, de $2 \mathrm{~cm}$ de diámetro, glabros o con pubescencia concentrada hacia los nudos; hojas opuestas; pecíolos de 8-10 cm de largo, angostamente alados; láminas foliares pinnadas o bipinnadas, $11-30 \mathrm{~cm}$ de largo y $10-15 \mathrm{~cm}$ de ancho, estipelas a veces presentes; 3-7 folíolos ovado lanceolados, sésiles o con peciólulos cortos, de 6-13 cm de largo y $0.5-7 \mathrm{~cm}$ de ancho, agudos en el ápice, atenuados en la base, margen muy aserrado con 2-8 dientes por lado, de textura membranácea, de color verde pardo y pubescentes en el haz, verde pálido y pubescentes a lo largo de las nervaduras en el envés; cabezuelas en grupos de 2-4, de 8-12 cm de diámetro, varias sincrónicas; pedúnculos de 5-22 cm de largo; involucro campanulado; filarias externas 5 , ovadas a oblongas o espatuladas, 9-12 $\mathrm{mm}$ de largo y $3-4.5 \mathrm{~mm}$ de ancho, verdes, el margen púrpura, reflejas en antesis; filarias internas 8 , elípticas a ovadas u oblongas, $1.5-2 \mathrm{~cm}$ de largo, verdes amarillentas, glabras y con 5-12 nervaduras; páleas similares a las filarias internas; flores liguladas 8 , estériles, tubos pilosos, lígulas elípticas a ovadas, $2.5-4.5 \mathrm{~cm}$ de largo y $1.8-2.2 \mathrm{~cm}$ de ancho, violadas; flores del disco 56-68, tubulares, 7-12 mm de largo y 2-3 mm de ancho, amarillas, lóbulos extendidos o reflejos; anteras de 3-4 mm de largo, de color de café; ramas del estilo oblanceoladas, agudas y pubérulas en su parte abaxial y distal; aquenios linear oblanceolados, 7-15 $\mathrm{mm}$ de largo y 2-3 $\mathrm{mm}$ de ancho, grises; vilano ausente; receptáculo plano, 6.5-7 $\mathrm{mm}$ de diámetro.

Comentarios taxonómicos. Dahlia sherffii se conocía de la vertiente este de la sierra Madre Occidental en los estados de Chihuahua y Durango. Aquí damos cuenta de su presencia en el norte de Jalisco, región que constituye el extremo sur de la misma sierra. Prospera en laderas rocosas en sitios abiertos del bosque de pino y encino, en altitudes que oscilan entre 2400 y $2700 \mathrm{~m}$. Las poblaciones de $D$. sherffii en Chihuahua y Durango se componen de numerosos individuos y en consecuencia se considera sin problemas de supervivencia.

Material estudiado. México. Jalisco: municipio de Huejuquilla El Alto: San Andrés Cohamiata, J. A. Bauml 1638 (RSA).

Dahlia tenuicaulis P. D. Sorensen, Rhodora 71: 335. 1969 (Fig.1D).

Arbusto decumbente o de aspecto arborescente, 2-4.5 m de altura; raíces tuberosas; tallos lignificados, de $3-7 \mathrm{~cm}$ de diámetro, glabros; hojas opuestas; pecíolos de $8-15 \mathrm{~cm}$ de largo; láminas foliares elípticas a oblongas en contorno general, 30-45 cm de largo y 10-15 cm de ancho, pinnadas o bipinnadas; folíolos 3-7, lanceolados, con peciólulos de 2-8 cm de largo, acuminados en el ápice, atenuados en la base, margen aserrado con 8-14 dientes por lado, de textura membranácea, de color verde pardo en el haz, verdes pálidos y pubescentes a lo largo de las nervaduras en el envés; cabezuelas en grupos de 4-8, 8-15 cm de diámetro, varias sincrónicas; pedúnculos de 10-22 cm de largo; involucro campanulado; filarias externas 5-6, ovadas a romboides, 9-12 mm de largo y 4-6 mm de ancho, verdes, abiertas en antesis; filarias internas 8, lanceoladas u oblongas, 1.5-2.5 $\mathrm{cm}$ de largo, amarillas pálidas, glabras; páleas similares a las filarias internas aunque de dimensiones menores; flores liguladas 8 , estériles, tubo glandular pubescente; lígulas elípticas, $3-5 \mathrm{~cm}$ de largo y $1.8-2.5 \mathrm{~cm}$ de ancho, violadas; flores del disco 70-80, tubulares, 8-12 $\mathrm{mm}$ de largo, amarillas, lóbulos erectos; anteras de 3-5 $\mathrm{mm}$ de largo, de 
color amarillo anaranjado; ramas del estilo oblanceoladas, agudas y pubérulas en la parte distal; aquenios linear oblanceolados, $11-15 \mathrm{~mm}$ de largo y 2-3 $\mathrm{mm}$ de ancho, grises; vilano ausente; receptáculo plano, $10-14 \mathrm{~mm}$ de diámetro.

Comentarios taxonómicos. Dahlia tenuicaulis tiene amplia distribución a lo largo de la vertiente pacífica del país, sin embargo, al menos en Jalisco, Colima y Guerrero sus poblaciones tienen pocos individuos (Cuadro 1). Crece en bosques de pino, de pino y encino y con mayor frecuencia en bosques de abeto. En Jalisco se conoce en pocas localidades ubicadas en el sur y suroeste del estado, en altitudes que van desde los 2600 y hasta los $3500 \mathrm{~m}$. Florece de agosto a octubre.

Material estudiado. México. Jalisco: municipio de Autlán de Navarro: puerto Los Mazos, 15.XI.1994, flor y fruto, J. J. Reynoso-Dueñas et al. 2782 (IBUG). Municipio de Ayutla: Km. 5 de la brecha El Rosario-La Leona, 7-8 $\mathrm{km}$ al sur de San Miguel de la Sierra, 10.X.2010, flor y fruto, A. Castro-Castro y J. L. Villa 2301 (IBUG, IEB, MEXU). Municipio de Casimiro Castillo: puerto Los Mazos, por la carretera a Autlán, 24.IX.1988, O. Reyna 712 (IBUG). Municipio de Concepción de Buenos Aires: terracería a Concepción de Buenos Aires, 13.IX.1976, flor, L. M. Villareal de Puga et al. 13714 (IBUG). Municipio de Cuautitlán de García Barragán: $20 \mathrm{~km}$ South of El Chante, 22.IX.1978, flor, H. H. Iltis et al. 78 (IBUG, WIS). Municipio de Tapalpa: camino entre Juanacatlán y Tepec, 23.VIII.1986, flor, A. Rodríguez y J. Suárez 584 (IBUG). Municipio de Tolimán: Nevado de Colima, 30.XII.1983, flor y fruto, L. M. González-Villareal 1932 (IBUG). Municipio de Tonila: Volcán de Fuego, camino al alberge, 14.VI.1986, flor, L. M. González-Villarreal y J. A. Pérez de la Rosa 2508 (IBUG, IEB). Municipio de Tuxpan: $13 \mathrm{~km}$ al sur de La Mesa, brecha al parque nacional El Nevado, 5.I.1990, flor y fruto, J. Villa y J. Chávez 514 (IBUG). Municipio de Venustiano Carranza: brecha Venustiano Carranza-Tapalpa, 17.IX.1988, flor, L. Vigueras et al. 75 (IBUG). Municipio de Zapotitlán de Vadillo: ladera de exposición norte del Nevado de Colima, 14.X.1984, flor. R. González-Tamayo s.n. (IBUG). Municipio de Zapotlán el Grande: Nevado de Colima, brecha El Fresnito a El Colimote, 19.X.1988, flor. M. Cházaro 5736 (IBUG).

Dahlia pugana Aaron Rodr. et A. Castro sp. nov. Tipo: MÉXICO: JALISCO: municipio de Zapopan, cerro El Tepopote, cañadas que desaguan al sur del cerro, frente al poblado La Primavera, $1900 \mathrm{~m}$ alt. 6.VIII.2009. A. CastroCastro et A. Rodríguez 1847 (holótipo: IBUG; isótipos: IEB, MEXU (Figs. 1E-F, 2).

Dahlia pugana Aaron Rodr. et A. Castro D. barkerae Knowles et West., D. brevi P. D. Sorensen et D. scapigerae (A. Dietr.) Knowles et West. similia herba pilosa simplicaule internodia 2-5 cm stipellis destitute phyllaria externa erecta vel pauco expansa flores ligulatis sterilis flores disci 20-55 in numero achaeniis dimorphis radii obovatis $4 \mathrm{~mm}$ longis achaenis centri elipticis $8 \mathrm{~mm}$ longis muricatis pappus disciformis differ.

Herbácea perenne de $40-70 \mathrm{~cm}$ de alto, con el follaje concentrado en la parte basal; raíces tuberosas, fasciculadas, fusiformes; tallo fistuloso, estriado, simple, pubescente sobre todo en los nudos, $2.5-5 \mathrm{~mm}$ de diámetro, entrenudos de (2) 3-4 (5) cm de longitud; hojas opuestas, elípticas a ovadas, pinnadas, bipinnadas o rara vez enteras, (4.5) 9-14 (17) cm de largo y (3) 4.5-7 (9) cm de ancho; pecíolo sólido, a veces con alas angostas sobre todo en la porción distal, (2.5) 3.5-5.5 (7.5) cm de largo, sin estipelas; folíolos 3-5 (7), opuestos, distantes entre sí 2-3.5 cm, sésiles y a veces con peciólulos de hasta $5 \mathrm{~mm}$ de largo, oblanceolados, romboides u oblongos, (1.5) 3.5-4 (5.5) $\mathrm{cm}$ de largo y (0.5) 1.4-2 (2.2) $\mathrm{cm}$ de ancho, base cuneada pero con frecuencia oblicua, agudos a acuminados en el ápice, margen entero o subentero con 1-4 dientes de cada lado y ciliolado, membranáceos, verde oscuro, muricados e híspidos en el haz, notablemente más pálidos e hispídulos en el envés, los tricomas multicelulares duros de 1-1.5 mm de longitud; foliolo terminal de igual o poco mayor tamaño que los laterales; cabezuelas 3 , rara vez solitarias, $5.5-6 \mathrm{~cm}$ de diámetro; pedúnculos de $20-50 \mathrm{~cm}$ de largo de color púrpura pardo; involucro campanulado a subhemisférico, glabro, filarias externas 5, erectas o poco extendidas en antesis, lanceoladas, gibosas en la mitad, 5-7 $\mathrm{mm}$ de largo y 1.5-2.5 $\mathrm{mm}$ de ancho, crasas, verdes, con 3-5 nervaduras marcadas de color púrpura; filarias internas 8 (10), lanceoladas, $1.2-1.5 \mathrm{~cm}$ de largo y $4-4.5 \mathrm{~mm}$ de ancho, agudas a redondeadas en el ápice y truncadas en la base, verde translúcido en su inserción al receptáculo, membranáceas, con 8-12 nervaduras marcadas de color púrpura; páleas similares a las filarias interiores; flores liguladas 8, estériles, tubos pilosos, sus láminas ovadas a elípticas, 2.3-2.9 $\mathrm{cm}$ de largo y 1-1.4 cm de ancho, violadas, con 2-4 nervaduras paralelas bien marcadas de color púrpura, tricomas multicelulares en su mayoría concentrados sobre las nervaduras y pocos dispersos sobre la lámina; flores del disco 20-55, corolas tubulares, 6.5-8 $\mathrm{mm}$ de largo y $1.5-2.5 \mathrm{~mm}$ de ancho en antesis, amarillas; tubo de 2-2.5 mm de largo y $0.5 \mathrm{~mm}$ de ancho, con tricomas dispersos en la base, lóbulos erectos o un tanto extendidos; anteras con ápices lanceolados y bases auriculadas, 4-5 $\mathrm{mm}$ de largo, de color de café; ramas del estilo abiertas en antesis, lanceoladas, agudas y densamente ciliadas; aquenios dimórficos; los del margen del receptáculo obovados, $4 \mathrm{~mm}$ de largo y $2 \mathrm{~mm}$ de ancho; los del centro del receptáculo elípticos, $8 \mathrm{~mm}$ de largo y $2 \mathrm{~mm}$ de ancho; ambos tipos cóncavos en su cara adaxial, convexos en 


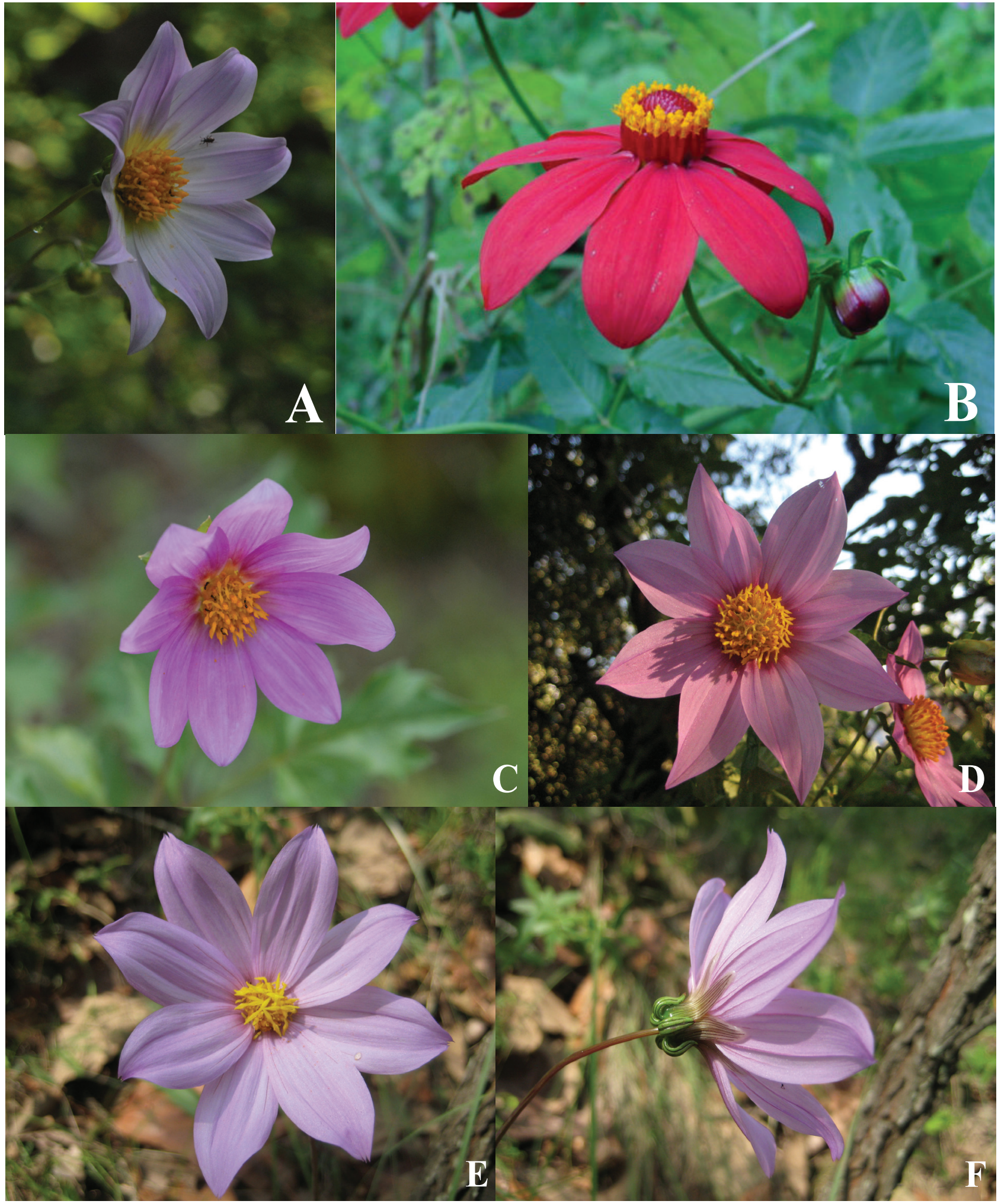

Figura 1. Las dalias del estado de Jalisco. A, Dahlia barkerae; B, D. coccinea; C, D. sherffii; D, D. tenuicaulis; E, D. pugana, vista frontal de la cabezuela; F, D. pugana, vista lateral y posición de las filarias. 


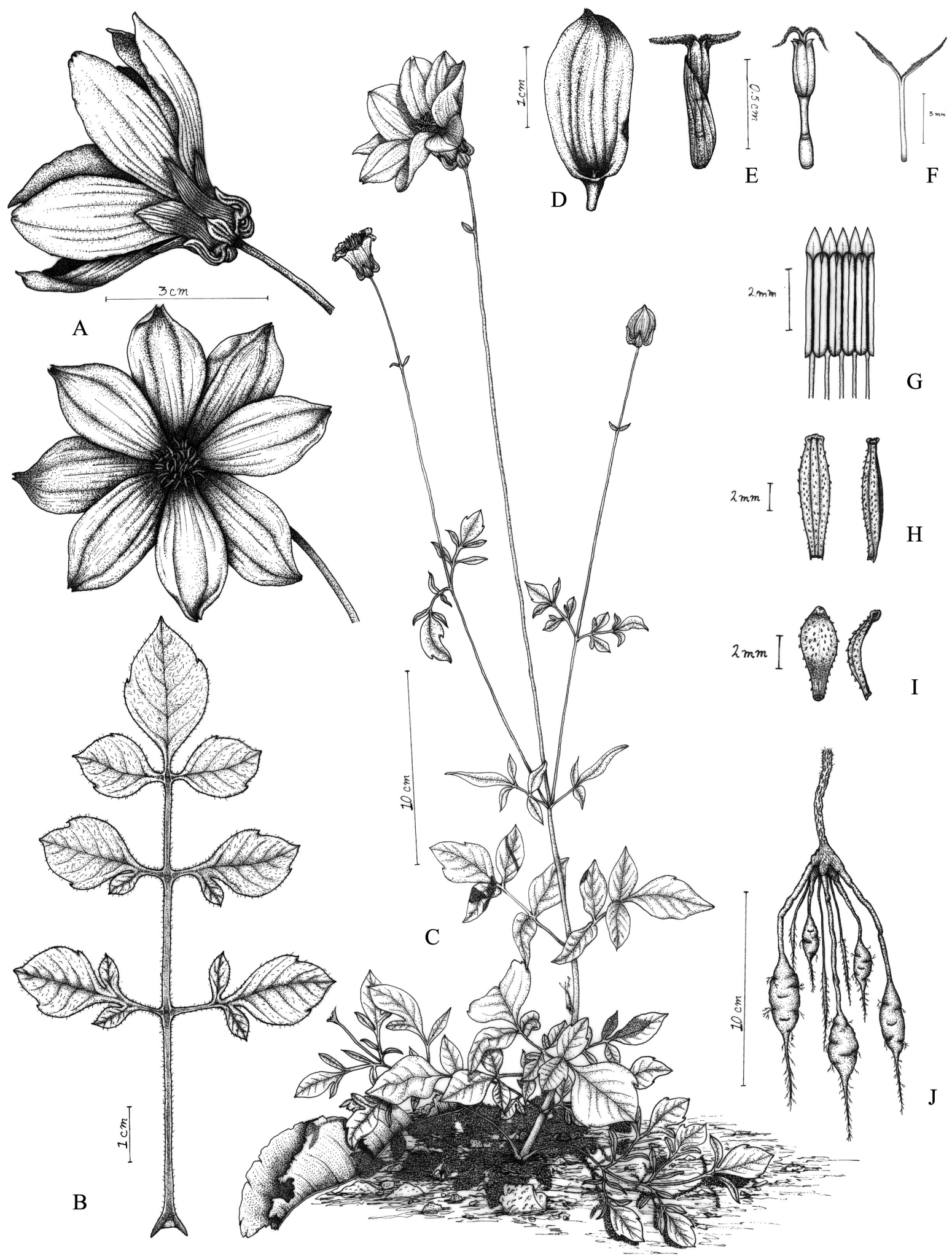

Figura 2. Dahlia pugana. A, cabezuelas; B, hoja madura; C, aspecto general; D, flor ligulada; E, flores del disco; F, estilo; G, anteras; H, I, aquenios; J, raíces (A. Castro-Castro y A. Rodríguez 1847, IBUG). 
su cara abaxial, muricados, negruzcos, pubérulos; vilano disciforme; receptáculo convexo de 6.5-8 $\mathrm{mm}$ de diámetro. Resumen taxonómico

Etimología. Dedicamos la especie en honor a la maestra Luz María Villareal de Puga, fundadora del herbario que lleva su nombre en el Instituto de Botánica de la Universidad de Guadalajara (IBUG). La maestra Puga ha generado una gran colección de la flora de la Nueva Galicia y ha sido una incansable formadora de botánicos. Un ejemplar, recolectado por ella en 1968, inspiró la búsqueda de más y la descripción de la especie.

Fenología. Dahlia pugana florece desde finales de julio hasta principios de septiembre y fructifica durante septiembre. Su floración no se traslapa con la de $D$. coccinea, especie con la cual cohabita. Su breve periodo de floración al inicio de la temporada de lluvias y la reducida producción de cabezuelas por individuo, pudieron haber sido motivos de su pobre representación en los herbarios.

Distribución, hábitat y conservación. Dahlia pugana crece en el centro de Jalisco. Se conoce de un área de $8 \mathrm{~km}^{2}$ en el municipio de Zapopan. Crece en un intervalo altitudinal entre 1800 y 1950 m. Habita en cañadas húmedas de exposición noreste y suelos delgados y ácidos en el bosque mixto de encino y pino. Como especies asociadas destacan Quercus coccolobifolia Trel., Q. praineana Trel., $Q$. resinosa Liebm., $Q$. viminea Trel., Pinus oocarpa Schiede ex Schltdl., Clethra rosei Britton, Befaria mexicana Benth., Vaccinium stenophyllum Steud., Cosmos landii Sherff var. achalconensis Melchert, Prochnyanthes mexicana (Zucc.) Rose, Calliandra hirsuta (G. Don) Benth., Pitcairnia karwinskyana Schult. et Schult. f., Styrax jaliscana S. Watson, Ximenia parviflora Benth., Schoenocaulon jaliscense Greenm., Salvia angustiarum (A. Gray ex S. Watson) Epling, S. heterotricha Fernald y Randia capitata DC. Dahlia pugana es conocida sólo de su localidad tipo. La presión humana, con fines inmobiliarios, a que está sujeta el área, convierten a D. pugana en una especie vulnerable. Parátipos. México. Jalisco: municipio de Zapopan: cerro El Tepopote, $30 \mathrm{~km}$ al oeste de Guadalajara, 4.VIII.1968, flor, L. M. Villareal de Puga 1662 (IBUG); cerro El Tepopote, subiendo por las faldas que ven a la carretera a Nogales, 6.X.2009, fruto, A. Castro-Castro y A. Frías 1870 (IBUG, MEXU); cima del cerro El Tepopote, vereda de ascenso a la antena, al oeste de Nextipac, 27.VII.2010, flor, A. Castro-Castro y J. González 2177 (IBUG, MEXU, IEB); cerro El Tepopote, vereda de ascenso a la antena y bajando por la cara de exposición norte, por el antiguo camino a Tequila, 28.VIII.2010, flor y fruto, A. CastroCastro et al. 2209 (IBUG, MEXU, IEB); faldas del cerro El Tepopote con exposición este, 11.IX.2010, fruto, $A$. Frías et al. 1760 (IBUG).
Comentarios taxonómicos. Dahlia pugana tiene tallos herbáceos, pinnas opuestas sobre las raquillas, pecíolos sólidos y filarias externas erectas, caracteres que permiten ubicarla en Dahlia sección Dahlia (Sorensen, 1969; Hansen, 2004). La especie nueva es morfológicamente afín a $D$. brevis P. D. Sorensen, $D$. barkerae y $D$. scapigera (A. Dietr.) Knowles et Westc. (Cuadro 2). Este grupo de especies se caracteriza por sus lígulas violadas, que de acuerdo a la clasificación Methuen handbook of colour (Kornerup y Wanscher, 1978) corresponde a violeta (1617) A (4-2).

Dahlia pugana es diferente a $D$. brevis por tener folíolos siempre opuestos, de bases oblicuas y pecíolos más largos. Además, sus aquenios son elípticos u obovados y pubérulos. El tamaño de la cabezuela y el receptáculo es más reducido en D. pugana que en D. brevis. Una característica contrastante entre ambas especies es el tallo. En $D$. pugana es simple pero en $D$. brevis es muy ramificado (Cuadro 2). A diferencia de Dahlia pugana, D. barkerae tiene hojas estipeladas y sus flores liguladas son fértiles. En D. pugana sólo existe un tallo, mientras que $D$. barkerae tiene varios. Más aún, las cabezuelas son solitarias en D. pugana, en contraste con $D$. barkerae que presenta múltiples cabezuelas sincrónicas. Por último, D. scapigera es diferente a $D$. pugana por su hábito escaposo y la presencia de estipelas en sus hojas; además, en D. pugana las flores líguladas son estériles, en tanto que en D. scapigera son fértiles. También, los taxones comparados difieren en sus intervalos de distribución latitudinal y altitudinal y en sus preferencias ecológicas.

\section{Agradecimientos}

A Osvaldo Zuno, por la elaboración de la ilustración; a Pablo Carrillo Reyes, por las excelentes aportaciones al manuscrito; a Roberto González Tamayo, por su ayuda en la revisión de la diagnosis en latín, y a dos revisores anónimos, por las valiosas sugerencias. De manera especial, a Guadalupe Munguía Lino, Virginia Ramírez Cruz, Alfredo Frías Castro, Esteban Suárez Muro, Jesús G. González Gallegos, Jesús Padilla Lepe y José Luis Villa Vázquez, por su apoyo durante el trabajo de campo; a Hans V. Hansen, Gregorio Nieves y Fidel Landeros, por facilitarnos diversas referencias bibliográficas, y a Mariana Rascón Montaño, quien elaboró la base de datos de los ejemplares del herbario IBUG. Asimismo, a los curadores de los herbarios CIIDIR, CIMI, ENCB, GUADA, IBUG, IEB, MEXU, WIS y ZEA, por las facilidades concedidas durante la consulta de sus colecciones.

El trabajo fue financiado por el Consejo Nacional de Ciencia y Tecnología (CONACYT) a través del proyecto 80200, convocatoria CB-2007-01. 
Cuadro 2. Principales diferencias morfológicas y geográficas entre Dahlia pugana, D. barkerae, D. brevis y D. scapigera

\begin{tabular}{|c|c|c|c|c|}
\hline Carácter & D. pugana & D. barkerae & D. brevis & D. scapigera \\
\hline Hábito & $\begin{array}{l}\text { Hierba de } 40-70 \mathrm{~cm}, \text { sin } \\
\text { ramificarse }\end{array}$ & $\begin{array}{l}\text { Hierba de } 90-150 \mathrm{~cm}, \\
\text { ramificada }\end{array}$ & $\begin{array}{l}\text { Hierba de } 40-70 \mathrm{~cm}, \\
\text { muy ramificada }\end{array}$ & $\begin{array}{l}\text { Hierba de } 60-90(180) \\
\mathrm{cm}, \text { sin ramificarse }\end{array}$ \\
\hline Entrenudos & (2) $3-4(5) \mathrm{cm}$ & $0-1(6.5-14) \mathrm{cm}$ & $4-8 \mathrm{~cm}$ & $0-1(6-10) \mathrm{cm}$ \\
\hline Hoja & $\begin{array}{c}\text { Enteras, pinnadas, } \\
\text { bipinnadas, (4.5) 9-14 } \\
\text { (17) } \mathrm{cm}\end{array}$ & $\begin{array}{l}\text { Enteras, pinnadas o } \\
\text { trifoliadas, } 16-30 \mathrm{~cm}\end{array}$ & $\begin{array}{l}\text { Pinnadas, pinatisectas, } \\
\text { bipinnadas, } 3.5-14 \mathrm{~cm}\end{array}$ & $\begin{array}{l}\text { Pinnadas, trifoliadas o } \\
\text { enteras, } 15-30 \mathrm{~cm}\end{array}$ \\
\hline Foliolo terminal & $\begin{array}{l}\text { De igual o poco mayor } \\
\text { tamaño que los laterales }\end{array}$ & $\begin{array}{c}\text { Más largo que los late- } \\
\text { rales }\end{array}$ & $\begin{array}{c}\text { Más pequeño que los } \\
\text { laterales }\end{array}$ & $\begin{array}{l}\text { Más largo que los late- } \\
\text { rales }\end{array}$ \\
\hline $\begin{array}{l}\text { Segmentación de las } \\
\text { hojas }\end{array}$ & 3-5 (7) segmentos & (3) 5-7 segmentos & 3-5 segmentos & (3) 5-7 (9) segmentos \\
\hline Estipelas & Ausentes & Presentes & Ausentes & Ausentes \\
\hline Pecíolo & (2.5) $3.5-5.5(7.5) \mathrm{cm}$ & $2.5-6(14) \mathrm{cm}$ & $0.7-2.2 \mathrm{~cm}$ & $6-15 \mathrm{~cm}$ \\
\hline Cabezuela & $\begin{array}{l}\text { (1) } 3 \text {, solitarias, } 5.5-6 \\
\mathrm{~cm} \text { de diámetro }\end{array}$ & $\begin{array}{c}\text { 2-3 (5), sincrónicas, } \\
\text { 4.5-5.5 cm de diámetro }\end{array}$ & $\begin{array}{l}\text { (1) 3-5 (8), sincrónicas, } \\
5.5-7.5 \mathrm{~cm} \text { de diámetro }\end{array}$ & $\begin{array}{c}1-3(6) \text {, solitarias o } \\
\text { sincrónicas, } 5-7.5 \mathrm{~cm} \text { de } \\
\text { diámetro }\end{array}$ \\
\hline Pedúnculo & $20-50 \mathrm{~cm}$ & $5.5-13 \mathrm{~cm}$ & $10-35 \mathrm{~cm}$ & $35-55 \mathrm{~cm}$ \\
\hline Filarias externas & $\begin{array}{l}\text { Erectas o poco extendi- } \\
\quad \text { das, } 3-5 \text { nervadas }\end{array}$ & Erectas, 3-5 nervadas & Erectas, 5-9 nervadas & $\begin{array}{l}\text { Erectas o extendidas, } \\
\text { 3-5 nervadas }\end{array}$ \\
\hline Flores liguladas & $\begin{array}{c}\text { Estériles, 2-4 nervadas, } \\
\text { violadas }\end{array}$ & $\begin{array}{c}\text { Fértiles, } 2 \text { nervadas, } \\
\text { violadas }\end{array}$ & $\begin{array}{c}\text { Estériles, } 3-8 \text { nervadas, } \\
\text { violadas }\end{array}$ & $\begin{array}{l}\text { Fértiles, } 3-5 \text { nervadas, } \\
\text { violadas o blancas }\end{array}$ \\
\hline Flores del disco & $20-55$ & $35-60$ & $55-70$ & $35-60$ \\
\hline Aquenios & $\begin{array}{l}\text { Dimórficos, elípticos, } \\
\text { muricados, pubérulos }\end{array}$ & $\begin{array}{l}\text { Linear oblanceolados, } \\
\text { pubérulos }\end{array}$ & Espatulados, glabros & $\begin{array}{l}\text { Linear oblanceolados, } \\
\text { pubérulos }\end{array}$ \\
\hline Vilano & Disciforme & $\begin{array}{l}\text { Ausente o con dos } \\
\text { pequeños hombros }\end{array}$ & Ausente & $\begin{array}{l}\text { Ausente o con dos } \\
\text { pequeños hombros }\end{array}$ \\
\hline Receptáculo & $6.5-8 \mathrm{~mm}$ de diámetro & 7-9 mm de diámetro & 9-12 mm de diámetro & 6-10 mm de diámetro \\
\hline Floración & Julio a septiembre & Junio a septiembre & Agosto a septiembre & Julio a octubre \\
\hline Rango altitudinal & $1800-1950 \mathrm{~m}$ & $2000-2800 \mathrm{~m}$ & $2800-3100 \mathrm{~m}$ & $2400-3300 \mathrm{~m}$ \\
\hline Distribución & Jal & Jal, Mich & Méx, Mich & $\begin{array}{l}\text { D. F., Gto, Hgo, Méx, } \\
\text { Mich }\end{array}$ \\
\hline
\end{tabular}

\section{Literatura citada}

Bauml, J. A. 1994. Etnobotany of the Huichol people of Mexico. Tesis doctorado, The Claremont Graduate School, California. $265 \mathrm{p}$.

Crawford, D. J., M. Tadesse, M. E. Mort, R. T. Kimball y C. P. Randle. 2009. Coreopsideae. In Systematics, evolution, and biogeography of Compositae, V. A. Funk, A Susanna, T. F. Stuessy y R. J. Bayer (eds.). International Association for Plant Taxonomy, Vienna. p. 713-730.

Hansen, H. V. 2004. Simplified keys to four sections with 34 species in the genus Dahlia (Asteraceae-Coreopsideae). Nordic Journal of Botany 24:549-553.

Hansen, H. V. y P. D. Sorensen. 2003. A new species of Dahlia (Asteraceae, Coreopsideae) from Hidalgo State, Mexico. Rhodora 105:101-105.
Kornerup, A. y J. H. Wanscher. 1978. Methuen handbook of colour, third edition. Methuen, London. $252 \mathrm{p}$.

McVaugh, R. 1984. Compositae. Flora Novo-Galiciana: a descriptive account of the vascular plants of Western Mexico, vol 12. The University of Michigan Press, Ann Arbor. p. $1-1157$.

Rzedowski, J. y G. Calderón de Rzedowski. 2008. Compositae: tribu Heliantheae. II. Flora del Bajío y Regiones Adyacentes 157:67-344

Saar, D. E. 2002. Dahlia neglecta (Asteraceae, Coreopsideae), a new species from Sierra Madre Oriental, Mexico. Sida 20:593-596.

Saar, D. E. y P. D. Sorensen. 2000. Dahlia parvibracteata (Asteraceae, Coreopsideae), a new species from Guerrero, Mexico. Novon 10:407-410.

Saar, D. E. y P. D. Sorensen. 2005. Dahlia sublignosa (Asteraceae): 
a new species in its own right. Sida 21:2161-2167.

Saar, D. E., P. D. Sorensen y J. P. Hjerting. 2002. Dahlia spectabilis (Asteraceae, Coreopsideae), a new species from San Luis Potosí, Mexico. Brittonia 54:116-119.

Saar, D. E., N. O. Polans y P. D. Sorensen. 2003a. A phylogenetic analysis of the genus Dahlia (Asteraceae) based on internal and external transcribed spacer regions of nuclear ribosomal DNA. Systematic Botany 28:627-639.

Saar, D. E., P. D. Sorensen y J. P. Hjerting. 2003b. Dahlia campanulata and D. cuspidata (Asteraceae, Coreopsideae): two new species from Mexico. Acta Botanica Mexicana 64:19-24.

Sherff, E. E. 1946. Some new or otherwise noteworthy dicotyledonous plants. American Journal of Botany 33:499-510.
Sherff, E. E. y E. J. Alexander. 1955. Coreopsidinae. In Compositae-Heliantheae-Coreopsidinae. North America Flora II 2:45-59.

Sorensen, P. D. 1969. Revision of the genus Dahlia (Compositae, Heliantheae-Coreopsidinae). Rhodora 71:309-365, 367-416.

Sorensen, P. D. 1980. New taxa in the genus Dahlia (Asteraceae, Heliantheae-Coreopsidinae). Rhodora 82:353-360.

Sorensen, P. D. 1987. Dahlia congestifolia, section Entemophyllon (Asteraceae: Heliantheae, Coreopsidinae), new from Hidalgo, Mexico. Rhodora 89:197-203.

Villaseñor, J. L., P. Maeda, J. J. Colín-López y E. Ortiz. 2005. Estimación de la riqueza de especies de Asteraceae mediante exploración a partir de datos de presencia-ausencia. Boletín de la Sociedad Botánica de México 76:5-18. 